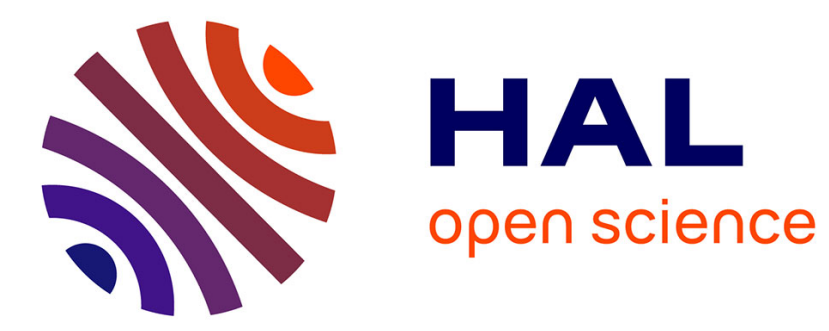

\title{
On the definability of radicals in supersimple groups
}

Cédric Milliet

\section{To cite this version:}

Cédric Milliet. On the definability of radicals in supersimple groups. The Journal of Symbolic Logic, 2013, 78 (2), pp.649-656. 10.2178/jsl.7802160 . hal-00680001v2

\section{HAL Id: hal-00680001 \\ https://hal.science/hal-00680001v2}

Submitted on 13 Apr 2012

HAL is a multi-disciplinary open access archive for the deposit and dissemination of scientific research documents, whether they are published or not. The documents may come from teaching and research institutions in France or abroad, or from public or private research centers.
L'archive ouverte pluridisciplinaire HAL, est destinée au dépôt et à la diffusion de documents scientifiques de niveau recherche, publiés ou non, émanant des établissements d'enseignement et de recherche français ou étrangers, des laboratoires publics ou privés. 


\title{
ON THE DEFINABILITY OF RADICALS IN SUPERSIMPLE GROUPS
}

\author{
CÉDRIC MILLIET
}

\begin{abstract}
If $G$ is a group with supersimple theory having finite $S U$-rank, the subgroup of $G$ generated by all of its normal nilpotent subgroups is definable and nilpotent. This answers a question asked by Elwes, Jaligot, Macpherson and Ryten in [5]. If $H$ is any group with supersimple theory, the subgroup of $H$ generated by all of its normal soluble subgroups is definable and soluble.
\end{abstract}

\section{INTRODUCTION}

Among the problems in the model theory of groups, is the one of knowing which subsets of a group $G$ are definable by a formula. For example, the centraliser of an element $a$ in $G$ is defined by the quantifier free formula $x a=a x$ and the centre of $G$ by the formula $(\forall y) x y=y x$. Similarly, finite sets, centralisers of finite sets and the iterated centres of $G$ are always definable. But this is mostly the end of the list: almost every other characteristic subgroups such as the commutator subgroup $G^{\prime}$, the $F C$-centre, the Fitting subgroup or the soluble radical may not be definable, not in first order logic at least: they all are countable union of definable sets. The situation is even more complicated for the iterated $F C$-centres, the $F C$-soluble radical or the $F C$-Fitting subgroup who have a higher complexity in the hierarchy of definable sets.

In an algebraic group over an algebraically closed field, every subgroup cited above is definable. The situation is far less straightforward in a group $G$ which is merely stable. Wagner has shown that the Fitting subgroup of $G$ is always definable [17]. The question is still open for the soluble radical of $G$, but Baudish [3] has proved that it is definable provided that $G$ be superstable. The starting point of their investigation was a Theorem of Poizat [13] that any nilpotent (respectively soluble) subgroup of $G$ is contained in a definable nilpotent (respectively soluble) one of same nilpotency class (resp. derived length). Recently, many attempts have been made to extend these results to a wider context: let us cite [15, Shelah] and [1, Aldama] for groups with dependent theory, [2, Altınel Baginski] for groups with the descending chain condition on centralisers, [11, Milliet] for groups with a simple theory and [5, Elwes Jaligot Macpherson Ryten], for supersimple groups, where it is shown that the soluble radical of a supersimple group $G$ of finite rank is definable and soluble provided that $G^{e q}$ eliminates $\exists^{\infty}$. The authors of [5] also asked whever such a $G$

2010 Mathematics Subject Classification. 03C45, 03 C60 (primary), 20F16, $20 \mathrm{~F} 18$ (secondary). Key words and phrases. Supersimple group, Fitting subgorup, soluble radical.

The paper arose after a conversation with Professor Macpherson in the Neostability theory conference in Banff, Canada. L'auteur voudrait remercier le centre de Banff pour son hospitalité, ainsi que l'institut Camille Jordan de Lyon qui lui a permis d'entreprendre ce long voyage. 
had a largest nilpotent normal subgroup $F$ and if $F$ would be definable. We give a positive answer here while proving that the Fitting subgroup of a supersimple group of finite $S U$-rank is definable and nilpotent. We also show that the soluble radical of a supersimple group of arbitrary rank is a definable and soluble subgroup. As a corollary, the $F C$-soluble radical of a supersimple group is virtually soluble and definable.

\section{Preliminaries on Groups}

If $G$ is a group and $x$ an element of $G$, we write $x^{G}$ for the conjugacy class $\left\{g^{-1} x g\right.$ : $g \in G\}$ of $x$, and $C(x)$ for its centraliser $\left\{g \in G: g^{-1} x g=x\right\}$ in $G$. If $y$ is another element of $G$, we write $[x, y]$ for the commutator $x^{-1} y^{-1} x y$. We write $G^{(n)}$ for the $n$th term of the derived series of $G$ defined inductively by puting $G^{(0)}$ equal to $G$ and $G^{(n+1)}$ the subgroup generated by the set $\left[G^{(n)}, G^{(n)}\right]$. The group $G$ is soluble of derived length $n$ if $n$ is the smallest natural number such that $G^{(n)}$ is $\{1\}$.

The $F C$-centre of a group $G$ is written $F C(G)$ and is defined to be the set of $g$ in $G$ such that $g^{G}$ is finite. By definition, the group $G$ is an $F C$-group if $F C(G)$ equals $G$. Inductively on $n$, we call $F C_{n+1}(G)$ the preimage in $G$ of $F C\left(G / F C_{n}(G)\right)$, with the convention that $F C_{0}(G)$ is $\{1\}$. This defines an ascending chain of characteristic subgroups of $G$. The group $G$ is called $F C$-nilpotent if $G$ equals $F C_{n}(G)$ for some natural number $n$, the least such we call the $F C$-nipotency class of $G$. Finite groups and nilpotent ones are both examples of $F C$-nilpotent groups. If $G / N$ is a quotient group modulo a normal subgroup $N$ of $G$, we write $F C_{G}(G / N)$ for the preimage of $F C(G / N)$ in $G$ by the canonical surjection from $G$ onto $G / N$.

Theorem 2.1 (Neumann [12]). An FC-group whose conjugacy classes are bounded by a natural number is abelian-by-finite and has a class 2 nilpotent subgroup of finite index.

Lemma 2.2. If $N$ is a finite normal subgroup of $G$, then $F C(G)$ equals $F C_{G}(G / N)$.

Proof. The canonical surjection from $G$ onto $G / N$ has a finite kernel. It follows that the conjugacy class $x^{G}$ is finite if and only if $(x N)^{G / N}$ is finite.

Lemma 2.3. If $H$ and $N$ are two normal subgroups of $G$ with $N \leq H$, then $F C_{G}(G / H) / N$ equals $F C_{G / N}(G / N / H / N)$.

Proof. There is a canonical homomorphism from $G / H$ onto $G / N / H / N$. It follows that $(x H)^{G / H}$ is finite if and only if $((x N) H / N)^{G / N / H / N}$ is finite. This means precisely that $x \in F C_{G}(G / H)$ if and only if $x N \in F C_{G / N}(G / N / H / N)$.

Lemma 2.4. If for some natural number $n$ the quotient $F C_{n+1}(G) / F C_{n}(G)$ is finite, then $F C_{n+2}(G)$ equals $F C_{n+1}(G)$.

Proof. We have

$$
F C_{n+2}(G) / F C_{n}(G)=F C_{G}\left(G / F C_{n+1}(G)\right) / F C_{n}(G)
$$

By Lemma 2.3

$$
F C_{n+2}(G) / F C_{n}(G)=F C_{G / F C_{n} G}\left(G / F C_{n}(G) / F C_{n+1}(G) / F C_{n}(G)\right)
$$


As $F C_{n+1}(G) / F C_{n}(G)$ is finite, applying Lemma 2.2 we get

$$
F C_{n+2}(G) / F C_{n}(G)=F C\left(G / F C_{n}(G)\right)=F C_{n+1}(G) / F C_{n}(G)
$$

Two subgroups of a given group $G$ are commensurable if the index of their intersection is finite in both of them. Commensurability is an equivalence relation on the set of subgroups of $G$.

Theorem 2.5 (Schlichting [16]). Let $G$ be a group and $H$ a subgroup of $G$ such that $H / H \cap H^{g}$ remains finite and bounded by a natural number for all $g$ in $G$. There exists a normal subgroup $N$ of $G$ such that $H / H \cap N$ and $N / N \cap H$ are finite. Moreover, $N$ is a finite extension of a finite intersection of $G$-conjugates of $H$. In particular, if $H$ is definable then so is $N$.

\section{Preliminaries on SUPERSIMPle Groups}

A supersimple group $G$ is equiped with a rank function taking values in the ordinals, and ranking any definable subset of $G$. We write $S U(X)$ for the rank of a definable subset $X$ of $G$. We shall not need the precise definition of the rank (we refer to [19] for more details), but only some of its properties that we recall now. The rank is increasing: if $X \subset Y$ are two definable subsets of $G$, then $S U(X)$ is smaller or equal to $S U(Y)$. If $G$ is supersimple, then so is any of its elementary extension, and so is $G^{e q}$, meaning that any quotient group $G / N$ by a definable normal subgroup $N$ has an ordinal $S U$-rank. A definable set (in $G^{e q}$ ) has rank zero if and only if it is finite. In particular, if $N$ is a definable normal subgroup of $G$, then $S U(G / N)$ equals zero if and only if $N$ has finite index in $G$.

The following comes from [6, Remark 3.5] as a particular case of [19, Theorem 5.5.4]

Theorem 3.1 (Zilber's Indecomposability Theorem). $G$ is a supersimple group of finite rank, $\left(X_{i}\right)_{i \in I}$ a family of definable subsets of $G$. There exists a definable subgroup $H$ of $G$ such that

(1) $H \leq\left\langle X_{i}: i \in I\right\rangle$

(2) Finitely many translates of $H$ cover $X_{i}$ for every $i$.

If the sets $X_{i}$ are normal in $G$, the group $H$ may be choosen normal in $G$.

Corollary 3.2. If $G$ is a supersimple group with finite $S U$-rank, then the derived subgroup $G^{\prime}$ is definable.

Proof. We follow exactly the proof of [8, Corollary 7.5]. Let $C$ be the set of commutators of $G$. By Theorem 3.1, there is a definable subgroup $H$ of $G^{\prime}$ with $H$ normal in $G$ such that finitely many translates of $H$ cover $C$. It follows that the set of commutators in $G / H$ is finite, so the derived group $(G / H)^{\prime}$ is finite by [9, p. 110]. The group $G^{\prime}$ is a finite union of cosets of $H$ hence definable.

Any ordinal $\alpha$ decomposes in base $\omega$ : there are unique ordinals $\alpha_{1}>\cdots>\alpha_{n}$ and non-zero natural numbers $k_{1}, \ldots, k_{n}$ such that $\alpha$ equals $\omega^{\alpha_{1}} \cdot k_{1}+\cdots+\omega^{\alpha_{n}} . k_{n}$. If $\alpha$ and $\beta$ are two ordinals, we may assume that $\alpha$ equals $\omega^{\alpha_{1}} \cdot k_{1}+\cdots+\omega^{\alpha_{n}} \cdot k_{n}$ and $\beta$ equals $\omega^{\alpha_{1}} \cdot \ell_{1}+\cdots+\omega^{\alpha_{n}} \cdot \ell_{n}$ for the same $\alpha_{1}, \ldots, \alpha_{n}$, adding some additional 
possibly zero $k_{i}$ and $\ell_{i}$ if necessary. We write $\alpha \oplus \beta$ for their Cantor sum defined by

$$
\alpha \oplus \beta=\omega^{\alpha_{1}} \cdot\left(k_{1}+\ell_{1}\right)+\cdots+\omega^{\alpha_{n}} \cdot\left(k_{n}+\ell_{n}\right)
$$

Theorem 3.3 (Lascar inequalities). $G$ is a supersimple group, and $H$ a definable normal subgroup of $G$. Then

$$
S U(H)+S U(G / H) \leq S U(G) \leq S U(H) \oplus S U(G / H)
$$

As a consequence, note that two definable subgroups of a supersimple group which are commensurable have the same $S U$-rank.

Proposition 3.4. $G$ is a supersimple group of rank $\omega^{\alpha_{1}} \cdot k_{1}+\cdots+\omega^{\alpha_{n}} \cdot k_{n}$ with $\alpha_{1}>\cdots>\alpha_{n}$. For every natural number $i$ such that $1 \leq i \leq n$, there is a definable normal subgroup $H$ of $G$ of rank $\omega^{\alpha_{1}} \cdot k_{1}+\cdots+\omega^{\alpha_{i}} \cdot k_{i}$. The group $H$ is unique up to commensurability.

Remark 3.5. Proposition 3.4 is the definable version of [18, Wagner, Corollary 4.2]. It generalises what is known for superstable groups [4, Corollary 2.7 p.27].

Proof. We may assume that $G$ is saturated and we write $\beta_{i}$ for $\omega^{\alpha_{1}} \cdot k_{1}+\cdots+\omega^{\alpha_{i}} \cdot k_{i}$. By [18, Corollary 4.2], there is a type-definable normal subgroup $H$ of $G$ having rank $\beta_{i}$. Recall that $\beta_{i}$ is by definition the rank of any of the generic types of $H$. By [18, Theorem 4.4], the group $H$ is the conjunction of definable groups $H_{i}$ for $i$ in $I$. We may close this familly by finite intersections, remove the members that do not have minimal rank and assume that every $H_{i}$ has rank $\beta$ say and are commensurable. It follows that for every $i$, the group $H$ has bounded index in $H_{i}$ so $H$ is a generic type of $H_{i}$ by [19, Lemma 4.1.15]. Thus $\beta$ equals $\beta_{i}$. Take any $H_{i}$. As $H$ is normal in $G, H_{i}^{g}$ and $H_{i}$ are commensurable for every $g$ in $G$. Let $F N\left(H_{i}\right)$ stand for the set of $g$ in $G$ such that $H_{i} / H_{i} \cap H_{i}^{g}$ is finite. On the one hand, the group $F N\left(H_{i}\right)$ is a countable union of definable sets. On the other hand, by [19, Lemma 4.1.15] and [19, Remark 4.1.5], it is type-definable. It must be definable by compactness and saturation. It follows that $H_{i} / H_{i} \cap H_{i}^{g}$ remains bounded by some natural number when $g$ ranges over $G$. By Theorem 2.5, there is a definable normal subgroup $N$ of $G$ commensurable with $H$ hence of rank $\beta_{i}$. If $K$ is another group satisfying the desired requirements, $K$ and $N$ are commensurable as type-definable sets by [18, Corollary 4.2] hence commensurable as definable sets.

Lemma 3.6 ([11]). If $G$ is a group with (super)simple theory, its FC-centre is definable.

Proof. It is shown in [11] that $F C(\bar{G})$ is definable by a formula $\psi$ provided that $\bar{G}$ be an $\aleph_{0}$-saturated extension of $G$. Actually the same formula $\psi$ computed in $G$ defines $F C(G)$.

\section{The Fitting SUbGroup}

Let $G$ be any group. We call the Fitting subgroup of $G$ the subgroup generated by all nilpotent normal subgroups. We write it $\operatorname{Fit}(G)$. It is worth mentioning that the Fitting subgroup is definable if and only if it is nilpotent. Namely $x$ belongs to Fit $(G)$ if and only if the subgroup generated by its conjugacy class $x^{G}$ is nilpotent. It follows that $\operatorname{Fit}(G)$ equals $\bigcup_{n \geq 1}\left\{x \in G:\left[x^{G} \cup\left(x^{-1}\right)^{G}, \ldots, n x^{G} \cup\left(x^{-1}\right)^{G}\right]=1\right\}$, 
where for any subset $X$ of $G$ the set $\left[X, \ldots,{ }_{n} X\right]$ is defined inductively by $\left[X,{ }_{1} X\right]=$ $\left\{\left[x_{1}, x_{2}\right]:\left(x_{1}, x_{2}\right) \in X^{2}\right\}$ and $[X, \ldots, n+1 X]=\{[y, x]:(y, x) \in[X, \ldots, n+1 X] \times X\}$. The observation that Fit $(G)$ is definable if it is nilpotent was first made by Ould Houcine, and the simple proof above was independently provided by the referee of [2].

Proposition 4.1. $G$ is a group and $F$ is a normal subgroup of $G$. Assume that $F \leq F C_{n}(G)$ for some natural number $n$ (in particular, $F$ is $F C$-nilpotent). If $G / F$ is $F C$-nilpotent, then so is $G$.

Proof. Assume that $G / F$ is $F C$-nilpotent of class $m$. There is a surjection from $G / F$ onto $G / F C_{n}(G)$. As recalled in [11], the image of an $F C$-nilpotent group by a group homomorphism is $F C$-nilpotent. It follows that $G / F C_{n}(G)$ is $F C$-nilpotent of class at most $m$, so that $F C_{m+n}(G)$ equals $G$.

We recall Hall's criterion for nilpotence.

Theorem 4.2 (Hall [7]). $G$ is any group and $N$ is a normal subgroup of $G$. If $G / N^{\prime}$ and $N$ are nilpotent, then $G$ is nilpotent.

Two other proofs of Theorem 4.2 can be found in [14] and [10], with a bound on the nilpotency class of $G$ depending on the classes of $N$ and $G / N^{\prime}$ in [10]. Note that since $G / N^{\prime \prime} / N^{\prime} / N^{\prime \prime}$ and $G / N^{\prime}$ are isomorphic, a straightforward induction on the nilpotency class of $N$ reduces the proof to the case where $N$ is 2-nilpotent.

Proposition 4.3 ([11]). $G$ is a group with (super)simple theory. If $G$ is $F C$ nilpotent of class $n$, then $G$ has a definable normal subgroup of finite index which is nilpotent of class at most $2 n$.

Theorem 4.4 (Milliet [11]). $G$ is a group with (super)simple theory. If $N$ is a normal nilpotent subgroup of class $n$, then $N$ is contained in a normal definable nilpotent subgroup of class at most 3 n.

We can now answer the question asked in [5].

Theorem 4.5. $G$ is a supersimple group with finite $S U$-rank. The Fitting subgroup of $G$ is definable and nilpotent.

Proof. By Lemma 2.4, for big enough $n$, the quotient $F C_{n+1}(G) / F C_{n}(G)$ is either trivial or infinite. By Lascar's equality, there exists a natural number such that $F C_{n}(G)=F C_{n+1}(G)$. We call $\bar{G}$ the quotient group $G / F C_{n}(G)$ so that $\bar{G}$ has a trivial $F C$-centre. Let $\bar{F}$ be its Fitting subgroup.

Claim 1. We may assume that $F C(G)=\{1\}$. To prove Claim 1, we need just assume that $\bar{F}$ is definable and nilpotent and show that $F i t(G)$ is definable and nilpotent. Note that $\bar{F}$ is $F C$-nilpotent. Let $F$ be its pull-back in $G$ so that we have $F / F C_{n}(G)=\bar{F}$. The group $F$ is $F C$-nilpotent by Proposition 4.1. By Proposition $4.3, F$ has a definable subgroup of finite index which is nilpotent, so it must have a normal one $N$. It follows that $F$ contains a maximal normal (in $G$ ) nilpotent subgroup $H$ of finite index so that $H$ equals $F i t(G)$. Being a finite extension of $N, \operatorname{Fit}(G)$ is definable.

Claim 2. We may assume that $G$ has a definable normal 2-nilpotent subgroup. On the one hand, if every nilpotent normal subgroup of $G$ is abelian, then $\operatorname{Fit}(G)$ is 
abelian. In this case, by the remark made at the beginning of this section (or Theorem 4.4), Fit $(G)$ must be definable. On the other hand, if there is a non abelian nilpotent normal subgroup, then there is a definable one by Theorem 4.4. Call it $N$. The group $Z_{2}(N)$ has the required properties.

Conclusion. We proceed by induction on $S U(G)$. If $S U(G)$ is zero, $G$ is finite and so is Fit $(G)$. If $S U(G)$ equals $n+1$, by Claim 2, there is a normal nilpotent definable subgroup $N$ of $G$ of nilpotency class 2. By Corollary 3.2, the derived subgroup $N^{\prime}$ is definable. As $N^{\prime}$ is normal un $G$, it is infinite by Claim 1, so $S U\left(N^{\prime}\right) \geq 1$. By Lascar's equality, $S U\left(G / N^{\prime}\right) \leq n$, and we may apply the induction hypothesis to $G / N^{\prime}$. It follows that $F i t\left(G / N^{\prime}\right)$ is definable and nilpotent. Let $F$ be its preimage in $G$ so that we have $F / N^{\prime}=F i t\left(G / N^{\prime}\right)$. By Theorem $4.2, F$ is nilpotent. Thus $F$ equals $F i t(G)$.

Remark 4.6. If $G$ is supersimple or rank $\omega$, every definable normal subgroup is finite or of finite index in $G$ by Lascar inequalities, so Fit $(G)$ is definable by Theorem 4.4. If $G$ has rank $\omega+1$, every definable normal subgroup has rank $0,1, \omega$ or $\omega+1$ by Lascar inequalities so a similar proof as in Theorem 4.5 works.

\section{The SOluble RAdical}

We call the soluble radical of $G$ the subgroup generated by all soluble normal subgroups and write it $R(G)$. It is a locally soluble group.

We recall a simple and useful remark by Ould Houcine :

Lemma 5.1 (Ould Houcine). $G$ is any group. Its soluble radical is definable if and only of it is soluble.

Proof. An element $x$ belongs to $R(G)$ if and only if the subgroup generated by its conjugacy class $x^{G}$ is soluble. Note that $\left\langle x^{G}\right\rangle^{(1)}$ is generated by all commutators of the form $\left[a^{g}, b^{h}\right]$ where $a$ and $b$ equal $x$ or $x^{-1}$ and $g$ and $h$ range over $G$. Thus the following equality holds

$$
R(G)=\bigcup_{n \geq 1}\left\{x \in G:\left(x^{G} \cup\left(x^{-1}\right)^{G}\right)^{(n)}=1\right\}
$$

where for any subset $X$ of $G$ the set $X^{(n)}$ is defined inductively by

$$
X^{(1)}=\{[x, y]:(x, y) \in X \times X\} \quad \text { and } \quad X^{(n+1)}=\left(X^{(n)}\right)^{(1)}
$$

It follows that $R(G)$ is a countable union of increasing definable sets. By compactness, it is definable if and only if this chain is stationnary.

Theorem 5.2 (Milliet [11]). $G$ is a group with (super)simple theory, $S$ a normal soluble subgroup of derived length $n$. The group $S$ is contained in a definable soluble group of derived length at most $3 n$.

Theorem 5.3. The soluble radical of a supersimple group is definable and soluble.

Proof. We proceed by transfinite induction on the rank of $G$.

If $G$ has rank zero, then $G$ is finite and so is $R(G)$.

If $G$ has a non-monomial rank, there is a natural number $k>0$ and ordinals $\alpha$ and $\beta$ such that $S U(G)$ equals $\omega^{\alpha} . k+\beta$ with $0<\beta<\omega^{\alpha}$. By Proposition 3.4, there is 
a normal subgroup $H$ of $G$ having $S U$-rank $\omega^{\alpha} . k$. We show that the derived length of any normal soluble subgroup of $G$ is bounded by some natural number. This is a necessary and sufficient condition by Lemma 5.1. So let $S$ be a normal soluble subgroup of derived length $n$. By Lascar inequalities, both $S U(G / H)$ and $S U(H)$ are less than $S U(G)$ so we may apply the induction hypothesis to $H$ and $G / H$ and it follows that $S H / H$ and $S \cap H$ are soluble of derived length at most some natural number $m$ say. As $S H / H$ is isomorphic to $S / S \cap H$, we must have $S^{(m)} \subset S \cap H$, so $S^{(2 m)}=\{1\}$. It follows that $n$ is bounded by $2 m$, as desired.

If $G$ has a monomial rank, it is of the form $\omega^{\alpha} . k$. Either there is some $a$ in $R(G)$ with $a^{G}$ having $S U$-rank at least $\omega^{\alpha}$. By Theorem 5.2, $a^{G}$ is contained in a definable normal soluble group $S$. As the rank is increasing, $S$ must have $S U$-rank at least $\omega^{\alpha}$. In that case, either $S$ and $G$ have the same rank so $G$ is virtually soluble and we are done, or $S U(S)<S U(G)$. Then, by Lascar inequalities 3.3 we have $S U(G / S)<S U(G)$ and we may conclude that the derived length of any normal soluble subgroup of $G$ is bounded by induction hypothesis, as in the non-monomial case. One last case to deal with: we may have $S U\left(a^{G}\right)<\omega^{\alpha}$ for all $a$ in $R(G)$. As $a^{G}$ and $G / C(a)$ are in bijection it follows that $S U(G / C(a))<\omega^{\alpha}$ for all $a$ in $R(G)$. By Lascar inequalities, this is equivalent to say that $S U(G / C(a))$ is zero for all $a$ in $R(G)$. So $R(G)$ is a subgroup of the $F C$-centre of $G$ which is definable by Lemma 3.6 and virtually nilpotent of class 2 by Theorem 2.1. It follows that $R(G)$ is also virtually nilpotent of class 2 (and locally soluble) hence soluble. In any case, $R(G)$ is definable and soluble.

\section{The $F C$-SOluble RADICAL}

Definition 6.1 (Duguid, McLain). A group $G$ is $F C$-soluble if there exists a finite sequence of normal subgroups $G_{0}, G_{1}, \ldots, G_{n}$ of $G$ such that

$$
G_{0}=G \unrhd G_{1} \unrhd \cdots \unrhd G_{n}=\{1\}
$$

and such that $G_{i} / G_{i+1}$ is an $F C$-group for all $i$. We call the least such natural number $n$ the $F C$-solubility class of $G$, or its class.

If $N$ is a normal subgroup of $G$, then $G$ is $F C$-soluble if and only if $G / N$ and $N$ are $F C$-soluble.

We define the $F C$-soluble radical of a group to be the subgroup generated by every normal $F C$-soluble subgroup. This is a locally $F C$-soluble subgroup:

Lemma 6.2. $H$ and $K$ are two normal $F C$-soluble subgroups of a group $G$ of class $h$ and $k$. The product $H . K$ is FC-soluble of derived length at most $h+k$.

Proof. The quotient $H K / K$ is isomorphic to $H / H \cap K$. So $K$ and $H K / K$ both are $F C$-soluble.

Proposition 6.3 (Milliet [11]). A (super)simple FC-soluble group is virtuallysoluble.

Corollary 6.4. The FC-soluble radical of a supersimple group is definable and virtually-soluble.

Proof. By Theorem 5.3, $R(G)$ is supersimple so the quotient $G / R(G)$ is supersimple and has no non trivial normal soluble subgroup. Let us write it $\bar{G}$. By 
Proposition 6.3, an $F C$-soluble subgroup of $\bar{G}$ is virtually-soluble, hence finite, so every normal $F C$-soluble subgroup is contained in $F C(\bar{G})$. By Lemma 3.6 and Theorem 2.1, the group $F C(\bar{G})$ must be finite. Its preimage in $G$ contains every normal $F C$-soluble subgroup of $G$.

\section{REFERENCES}

[1] Ricardo de Aldama, A result on definable groups without the independence property, to appear in the Bulletin of Symbolic Logic.

[2] Tuna Altinel and Paul Baginski, Definable envelopes of nilpotent subgroups of groups with chain conditions on centralizers, to appear in the Transactions of the American Mathematical Society.

[3] Andreas Baudish, On superstable groups, Journal of the London mathematical Society 42 (1980) 452-463.

[4] Chantale Berline et Daniel Lascar, Superstable groups, Annals of Pure and Applied Logic 30 (1986) 1-43.

[5] Richard Elwes, Eric Jaligot, Dugald Macpherson and Mark Ryten, Groups in supersimple and pseudofinite theories, Proceedings of the London Mathematical Society 103 (2011) 1049-1082.

[6] Richard Elwes and Mark Ryten, Measurable groups of low dimension, Mathematical Logic Quarterly 54 (2008) 374-386.

[7] Philip Hall, Some sufficient conditions for a group to be nilpotent, Illinois Journal of Mathematics 2 (1958), 787-801.

[8] Ehud Hrushovski, Pseudo-finite and related structures, Model theory and applications (eds L. Bélair, Z. Chatzidakis, P. D'Aquino, D. Marker, M. Otero, F. Point and A. Wilkie) Quaderni di Matematica 11 (Caserta 2003).

[9] James Humphreys, Linear Algebraic Groups, Springer Verlag, New-York 1981.

[10] Evgenii Khukhro, $p$-automorphisms of finite $p$-groups, London Mathematical Society Lecture Note Series, Cambridge University Press, 1998.

[11] Cédric Milliet, Definable envelopes in groups with simple theory, preprint.

[12] Bernhard H. Neumann, Groups covered by permutable subsets, Journal of the London Mathematical Society 29 (1954) 236-248.

[13] Bruno Poizat, Groupes stables, 1987.

[14] Derek Robinson, A course in the theory of groups, Springer, 1996.

[15] Saharon Shelah, Dependent first order theories, continued, (Shelah 783), to be published in the Israel Journal of Mathematics.

[16] Günter Schlichting, Operationen mit periodischen Stabilisatoren, Archiv der Matematik 34 (1980) 97-99.

[17] Frank O. Wagner, Fitting subgroup of a stable group, Journal of Algebra 174 (1995) 599-609.

[18] Frank O. Wagner, Groups in simple theories. Logic Colloquium 2001, 440-467, Lecture Notes in Logic, 20 Association of Symbolic Logic, Urbana, IL, 2005.

[19] Frank O. Wagner, Simple Theories, Kluwer Academic Publishers, Dordrecht, NL, 2000.

Université Galatasaray

Faculté de Sciences et de Lettres

Département de Mathématiques

ÇiraĞan Caddesi n. 36

34357 Ortaköy, Istamboul, Turquie

E-mail address: milliet@math.univ-lyon1.fr 